ISSN 0103-9954

\title{
SORTIMENTOS DE Pinus elliottii Engelm PARA A REGIÃO DA SERRA DO SUDESTE DO ESTADO DO RIO GRANDE DO SUL - BRASIL
}

\section{ASSORTMENTS OF Pinus elliottii Engelm FOR THE REGION OF SERRA DO SUDESTE, STATE OF RIO GRANDE DO SUL-BRAZIL}

\author{
Ronaldo Drescher $^{1} \quad$ Paulo Renato Schneider ${ }^{2}$ César Augusto Guimarães Finger ${ }^{3}$
}

\section{RESUMO}

No presente trabalho foi estudada a forma do tronco de Pinus elliottii Engelm, para formação de sortimentos e confecção de tabelas para uso na região da Serra do Sudeste do Estado do Rio Grande do Sul. A forma do tronco foi ajustada com boa precisão estatística por um polinômio de $5^{\circ}$ grau, tendo como variáveis dependentes os diâmetros relativos $\left(d_{i} / d\right)$ e como variáveis independentes as alturas relativas $\left(h_{i} / h\right)$. A integração do polinômio permitiu estimar os volumes absolutos e relativos dos sortimentos, sendo estes classificados e expressos em percentagem do volume e em número de toras correspondentes. Para isso, foi considerado, para quantificar o volume total aproveitável da árvore, o diâmetro mínimo de $7 \mathrm{~cm}$ com casca e para o volume de toras para a serraria, o diâmetro de $14 \mathrm{~cm}$ com casca, ambos tomados na ponta fina da tora. Esses resultados deram origem a tabelas de sortimentos otimizadas para árvores individuais.

Palavras-chave: forma de tronco, sortimento, Pinus elliottii

\begin{abstract}
In the present work the assortments of the of Pinus elliottii Engelm, with a view to the formation of assortments and tables of asssortments for regional use in the Serra do Sudeste, State of Rio Grande do Sul were studied. Thus, the development of stem taper for the medium tree and for diameter class and the formation of assortments were studied. Furthermore tables of assortments for regional use were developed. The polynomial models of $5^{\text {th }}$ degree showed good adjustment and statistical precision, with relative diameter $\left(\mathrm{d}_{\mathrm{i}} / \mathrm{d}\right)$ as dependent variable and relative height $\left(\mathrm{h}_{\mathrm{i}} / \mathrm{h}\right)$ as independent variable. Its integration allowed to estimate absolute and relative volumes of the assortments which were classified and expressed in percentage of the volume and number of corresponding logs. Thus, in order to quantify commercial volume of the tree, the minimum diameter of $7 \mathrm{~cm}$ outside bark at the small end of the tree and for sawlog timber a diameter of $14 \mathrm{~cm}$ outside bark were considered. These results are integrated in tables of optimized assortments for individual trees.
\end{abstract}

Key Words: taper, assortment, Pinus elliottii

1. Engenheiro Florestal, M.Sc., pelo Programa de Pós-Graduação em Engenharia Florestal, Centro de Ciências Rurais, Universidade Federal de Santa Maria, CEP: 97105-900, Santa Maria. (RS). Bolsista do CNPq.

2. Engenheiro Florestal, Dr., Professor Titular do Departamento de Ciências Florestais, Centro de Ciências Rurais, Universidade Federal de Santa Maria, CEP: 97.105-900, Santa Maria. (RS). Pesquisador do CNPq.

3. Engenheiro Florestal, Dr., Professor Adjunto do Departamento de Ciências Florestais, Centro de Ciências Rurais, Universidade Federal de Santa Maria, CEP: 97.105-900, Santa Maria. (RS). Pesquisador do CNPq. 


\section{INTRODUÇÃO}

No Rio Grande do Sul, Pinus elliottii Engelm é uma espécie amplamente utilizada nos reflorestamentos e florestamentos. Essa espécie é de extrema importância para o setor madeireiro da região pelo seu rápido desenvolvimento, boa forma e qualidade da madeira.

Em geral, a forma das árvores possui uma variação muito grande dentro da floresta. Essa variação ocorre por causa da diminuição do diâmetro em direção ao topo da árvore, sendo conhecida como "taper", afetando diretamente o volume e variando com a espécie, idade, espaçamento entre árvores e condições do sítio.

Nos últimos anos, observa-se na literatura o crescente interesse em estimar o volume comercial até alturas definidas ou diâmetros na parte superior do tronco, com a finalidade de saber qual a porção do tronco que poderá ser utilizada para produtos específicos.

A utilização de equações de forma do tronco possibilita, além de descrever o perfil do tronco, obter, por integração, a estimativa do volume total e parcial com grande precisão, permitindo também a confecção das tabelas de sortimentos.

Tendo em vista a inexistência de informações detalhadas sobre o crescimento e produção no atual desenvolvimento florestal brasileiro, em algumas regiões ou empresas, pesquisas foram e estão sendo desenvolvidas para descrever o sortimento das árvores em pé, subsídio indispensável para a elaboração do plano de ordenamento florestal.

A falta de tabelas de sortimentos apropriadas que permitam a determinação rápida do estoque de madeira para os diferentes tipos de aproveitamento, tem sido uma das grandes dificuldades do manejo florestal, em especial na avaliação econômica de povoamentos florestais de Pinus na região.

Embora não exista uma única classificação de sortimento possível e válida para todas as empresas florestais, deve-se definir uma que se ajuste especificamente à situação da empresa de acordo com suas próprias peculiaridades de utilização da madeira.

Em vista disso, o presente trabalho foi desenvolvido com o objetivo de determinar a forma de tronco, formação de sortimentos e a confecção de tabelas de sortimentos, para Pinus elliottii Engelm, da Região da Serra do Sudeste do Estado do Rio Grande do Sul.

\section{REVISÃO BIBLIOGRÁFICA}

A variação da forma do tronco das árvores acontece em razão do meio ambiente, espécie, idade, manejo a que são submetidas e de suas características genéticas. Essa variação da forma do tronco ocorre tanto em florestas naturais como em plantadas (SILVA, 1974 e FINGER, 1992).

Segundo SILVA (1974), em povoamentos compostos por uma única espécie, árvores que vegetam na periferia do povoamento, isoladas ou em grandes espaçamentos, apresentam uma forma natural ou espontânea, da mesma maneira que se vegetassem isoladamente. Já aquelas que se encontram no interior do povoamento, cuja sobrevivência acontece pela competição de água, luz e 
nutrientes, adquirem uma forma reta com poucas ramificações e uma baixa dimensão de copa, conhecida como forma florestal típica.

Vários autores, como AHRENS (1982) e SCHNEIDER (1993), citam que as funções de forma do tronco atuam como ferramenta importante para predizer o diâmetro em qualquer ponto do tronco a partir de uma ou duas variáveis medidas. Essas funções passaram a ser utilizadas também para estimar o volume do povoamento e construir tabelas de volume e sortimento para diferentes limites de dimensões de toras impostos pelo mercado.

A técnica de modelagem, empregada para descrição do perfil de troncos, vem sendo utilizada com êxito sendo considerado que um modelo é compatível ou não, quando a estimativa do volume total, obtida por integração de segmentos do tronco, seja semelhante àquela dada pela equação do volume da qual a equação de forma foi derivada (MUNRO \& DEMAERSCHALK, 1974).

Alguns modelos estatísticos foram e são utilizados para expressar a forma do tronco de espécies florestais (BORGES (1981), KOEHLER (1982), SILVA (1982), FRIEDL (1989), MOURA (1994), FINGER et al. (1995), FIGUEREDO-FILHO et al. (1996) e SCHNEIDER et al. (1996)). Esses modelos possuem como variáveis, além do diâmetro à altura do peito, os diâmetros valendo-se das alturas relativas e a altura total.

SCHNEIDER (1984) estudou, para Pinus elliottii da Floresta Nacional de Passo Fundo, modelos de função de forma e obteve melhor ajuste dos dados com um polinômio de $5^{\circ}$ grau. Esse modelo teve um erro padrão de estimativa igual a 0,0579 e um coeficiente de determinação de 0,9653 .

A utilização de equações polinomiais foi proposta para diferentes espécies por vários autores, entre eles: BORGES (1981), KOEHLER (1982), SILVA (1982 e 1983), FRIEDL (1989), MOURA (1994), FINGER et al. (1995), MAINARDI (1995), FIGUEREDO-FILHO et al. (1996), SCHNEIDER et al. (1996). Essas equações possibilitam a descrição da forma com uma única função e são de fácil aplicação.

JORGE (1984) estudou e elaborou tabelas de sortimento individuais para povoamentos de Pinus elliottii Engelm, na Floresta Nacional de Três Barras, utilizando 150 árvores, abatidas em povoamentos com idades variando entre 14 e 20 anos. $O$ autor desenvolveu uma metodologia para estimar alturas, situada em qualquer parte do tronco entre a base e o ápice da árvore, por meio do uso das equações de forma.

O estudo de sortimento dos povoamentos florestais é de grande importância para a análise do potencial madeireiro, observando-se aspectos do planejamento das atividades de manejo, dimensionamento das unidades de processamento mecânico da madeira, bem como da comercialização de madeira serrada (JORGE \& LARA, 1993).

\section{MATERIAIS E MÉTODOS}

Os dados para o estudo foram provenientes de florestas equiâneas situadas na localidade de 
Cordilheira da Serra do Piquiri, no extremo sul do município de Cachoeira do Sul, na região denominada de Serra do Sudeste. Essas florestas de propriedade da empresa Todeschini S.A, são formadas por povoamentos homogêneos de P. elliottii Engelm, implantados em junho de 1982, com um espaçamento inicial de $3 \times 2$ metros, nos quais foram realizados desramas e desbastes.

O clima da região é do tipo $\mathrm{Cfb} / \mathrm{g}$, definido pela temperatura média do mês mais quente de $24,8{ }^{\circ} \mathrm{C}$ e do mês mais frio de $13,6^{\circ} \mathrm{C}$. As chuvas estão distribuídas por todos os meses do ano, sendo junho o mês mais chuvoso, com $166 \mathrm{~mm}$, e dezembro o mês de menor ocorrência de chuvas, com $94 \mathrm{~mm}$. A região apresenta um déficit hídrico médio de $85 \mathrm{~mm}$, distribuídos de dezembro a março. A umidade relativa média anual é de 81\% (MORENO, 1961).

A formação de geadas é frequiente, com uma média de 22 ocorrências por ano, e os ventos mais comuns são do sudeste e do leste, esse último predominante no mês de abril. A altitude média da região varia entre 100 e 500 metros e o relevo é ondulado, com formações de vales abertos.

Conforme LEMOS et al. (1973), o solo da área experimental faz parte da unidade de mapeamento Pinheiro Machado que se caracteriza por solos litólicos, bem drenados, de coloração escura, textura média, com percentagens elevadas das frações mais grosseiras (areia grossa e cascalho), tendo como material de origem granito.

Para a determinação dos sortimentos, inicialmente, procedeu-se à integração matemática da função de forma selecionada.

A integração forneceu o volume do tronco total ou parcial conforme especificado no limite de integração. Por esse processo, foi possível determinar o volume para qualquer porção do tronco conforme exemplificado a seguir para o polinômio do $5^{\circ}$ grau.

Com a integração da equação, entre zero $(0)$ até a altura $\left(\mathrm{h}_{\mathrm{i}}\right)$, obtém-se o volume absoluto do tronco da seguinte forma:

$$
V=K * \int_{0}^{h_{i}} Y^{2} d_{h_{i}}
$$

Em que: $K=\pi / 4 ; Y=$ equação de forma de tronco.

No cálculo dos volumes dos sortimentos utilizou-se a integração parcial da função de forma, desde a altura do toco até a altura na ponta mais fina da tora. $\mathrm{O}$ volume desses sortimentos foi conseguido mediante subtração do volume obtido até o final da seção e o volume obtido até o início da seção, resultando na equação:

$$
\begin{aligned}
& V t=K *\left(\int_{0}^{x_{2}} Y^{2} d_{x_{2}}-\int_{0}^{X_{1}} Y^{2} d_{x_{1}}\right) \\
& \text { Em que: } x_{1}=\text { altura no início da tora, em metros; } \\
& x_{2}=\text { altura no fim da tora, em metros; } \\
& Y=\text { função de forma do tronco; } \\
& V_{t}=\text { volume da tora, em metros cúbicos; } K=\pi / 4 \text {. }
\end{aligned}
$$

Ciência Florestal, v. 9, n. 2, 1999 
Os sortimentos de madeira foram determinados mediante de um programa elaborado em linguagem Basic e expressos em percentagem do volume e em número de toras, tendo sido considerados, na sua determinação, o diâmetro mínimo de $14 \mathrm{~cm}$ com casca tomado na ponta fina e os seguintes critérios:

a) S1 = madeira para serraria com comprimento de 2,8 metros;

b) S2 = madeira para serraria com comprimento de 2,0 metros;

c) S3 = madeira para serraria com comprimento de 1,3 metros.

As alturas $\left(h_{i}\right)$ foram estimadas por uma equação polinomial, tendo como variáveis dependentes as alturas relativas $\left(h_{i} / h\right)$ e como independentes os diâmetros relativos $\left(d_{i} / d\right)$.

O fuste aproveitável foi otimizado para formar, preferencialmente, toras com o maior comprimento e o restante do fuste classificados nas classes ligeiramente inferiores, de maior comprimento.

A parte do fuste, com diâmetro maior que $7 \mathrm{~cm}$ e inferior a $14 \mathrm{~cm}$ com casca, e toda a parte do fuste, que não possibilitou a formação de uma tora com os comprimentos definidos, foram consideradas como volume para celulose e energia.

A variação da altura para uma classe de diâmetro foi obtida por meio da relação altura em função do diâmetro, expressa pela equação:

$\mathrm{h}=\mathrm{DAP} .\left(\mathrm{b}_{0}+\mathrm{b}_{1} . \mathrm{DAP}\right)$

Em que: $h=$ altura total, em metros;

$D A P=$ diâmetro à altura do peito, em centímetros;

$b_{0}, b_{1}=$ coeficientes.

No presente estudo, foram testadas as equações de forma de tronco apresentadas na Tabela 1, sendo selecionado, como o modelo que melhor representava a forma de tronco, aquele que apresentasse a maior precisão e melhor ajuste estatístico em relação ao: coeficiente de determinação $\left(\mathrm{R}^{2}\right)$; coeficiente de variação $(\mathrm{CV})$ e teste $\mathrm{F}$, bem como apresentasse distribuição de resíduos uniforme e sem tendências.

O modelo selecionado, por esse processo, foi utilizado para calcular regressões independentes para cada uma das árvores cubadas e para o estudo do sortimentos. Em seguida, realizou-se estudos de modelagem por meio do pacote estatístico SAS(1993) com o seu procedimento "stepwise".

\section{RESULTADOS E DISCUSSÃO}

O ajuste dos modelos estatísticos para Tabela 1 está sumarizado na Tabela 2. Verifica-se 
TABELA 1: Modelos de equações testadas para descrever a forma do tronco de Pinus elliottii.

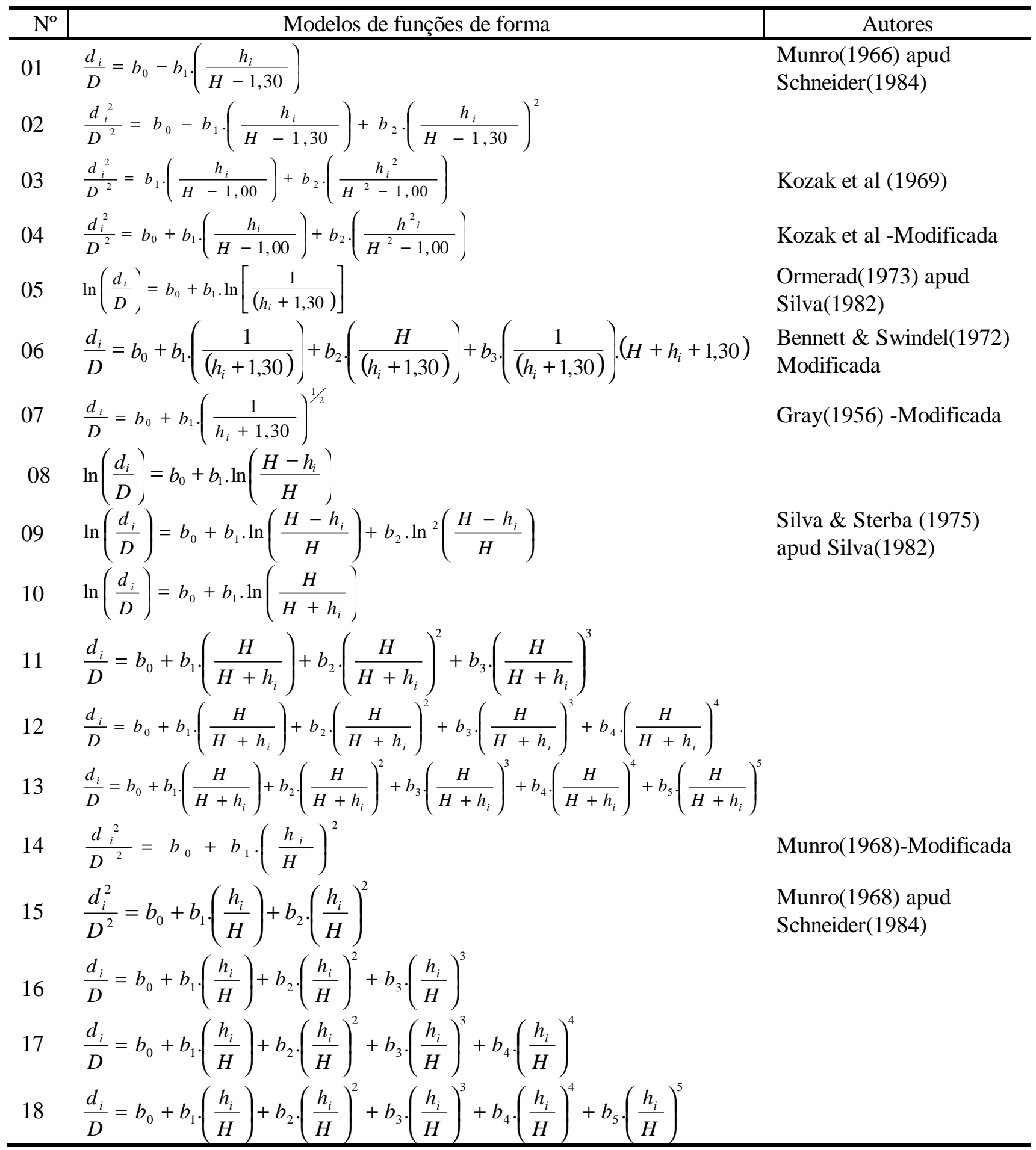

Sendo: $\mathrm{d}_{\mathrm{i}}=$ diâmetro à altura $\mathrm{i}$, em centímetros; $\mathrm{D}=$ diâmetro a altura do peito $(1,30)$, em centímetros; $\mathrm{h}_{\mathrm{i}}=$ altura na posição $i$, em metros; $H=$ altura total da árvore, em metros; $b_{0}, b_{1}, b_{2}, b_{3}, b_{4}$ e $b_{5}=$ coeficientes; ln = logarítimo natural.

Ciência Florestal, v. 9, n. 2, 1999 
que os modelos polinomiais do $3^{\circ}$ (modelo 16), $4^{\circ}$ (modelo 17) e $5^{\mathrm{o}}$ grau (modelo 18), com a variável independente $\left(\mathrm{h}_{\mathrm{i}} / \mathrm{h}\right)$, possuem os melhores ajustes, pois apresentaram, de forma conjunta, menor coeficiente de variação $(\mathrm{CV} \%)$ e maior coeficiente de determinação ajustado $\left(\mathrm{R}_{\text {aju}}^{2}\right)$, tendo também todos os coeficientes significativos, assim como a regressão (prob. >0,0001).

Estatisticamente, os melhores modelos foram os de número 17 e 18 . Esses dois modelos apresentaram os menores CV, iguais a $6,48 \%$ para o modelo 17 e $6,50 \%$ para o modelo 18 , e os maiores $\mathrm{R}_{\text {aju }}^{2} 0,97$ e 0,98 , respectivamente.

Os modelos 11, 12 e 13 também apresentaram boa precisão estatística. Os $\mathrm{R}_{\text {aju }}^{2}$ foram de 0,9678, 0,9694 e 0,9700, respectivamente. Seus coeficientes de variação foram: $6,50,6,48$ e 6,21, respectivamente.

Desses modelos, o polinômio de $5^{\circ}$ grau (modelo 18 ) foi selecionado para descrever a forma do tronco e, conseqüentemente, os sortimentos de árvores individuais, por apresentar boa precisão e por ter distribuição de resíduos mais uniforme quando comparado com os demais modelos testados.

TABELA 2: Análise estatística dos modelos de forma de tronco para Pinus elliottii.

\begin{tabular}{ccccc|c|c|c|c|r|r|r}
\hline Modelos & \multicolumn{1}{c|}{$\mathrm{b}_{0}$} & \multicolumn{1}{c}{$\mathrm{b}_{1}$} & $\mathrm{~b}_{2}$ & $\mathrm{~b}_{3}$ & $\mathrm{~b}_{4}$ & $\mathrm{~b}_{5}$ & $\mathrm{R}_{\text {aju }}^{2}$ & $\mathrm{~F}$ & Prob>F & $\mathrm{CV}(\%)$ \\
\hline 1 & 1,1820 & $-0,9297$ & & & & & 0,9477 & 11031,1 & 0,0001 & 9,53 \\
2 & 1,4116 & $-2,2916$ & 0,9682 & & & & 0,9131 & 3200,06 & 0,0001 & 20,03 \\
3 & 0,0000 & 3,6222 & $-4,4966$ & & & & 0,3411 & 158,86 & 0,0001 & 98,12 \\
4 & 1,4081 & $-2,3157$ & 1,1259 & & & & 0,9117 & 3143,56 & 0,0001 & 20,20 \\
5 & 0,5721 & 0,5558 & & & & & 0,6617 & 1192,11 & 0,0001 & $-91,23$ \\
6 & 0,1626 & 1,9043 & 0,0183 & 0,0000 & & & 0,8759 & 2150,71 & 0,0001 & 23,94 \\
7 & $-0,3657$ & 2,3288 & & & & & 0,9031 & 5673,84 & 0,0001 & 21,16 \\
8 & 0,1205 & 0,6730 & & & & & 0,9521 & 12116,8 & 0,0001 & $-34,31$ \\
9 & 0,1627 & 0,8192 & 0,0520 & & & & 0,9607 & 7451,53 & 0,0001 & $-31,08$ \\
10 & 0,3320 & 2,3196 & & & & & 0,8067 & 2542,74 & 0,0001 & $-68,96$ \\
11 & $-12,0950$ & 47,973 & $-60,1549$ & 25,5685 & & & 0,9678 & 8949,03 & 0,0001 & 6,50 \\
12 & 0,4624 & $-22,178$ & 84,1505 & $-104,031$ & 42,9141 & & 0,9694 & 7231,38 & 0,0001 & 6,48 \\
13 & $-47,116$ & 311,372 & $-838,383$ & 1154,43 & $-804,063$ & 225,0930 & 0,9700 & 5964,67 & 0,0001 & 6,21 \\
14 & 1,0941 & $-1,4762$ & & & & & 0,7382 & 1718,32 & 0,0001 & 34,77 \\
15 & 1,4095 & $-2,4979$ & 1,1499 & & & & 0,9131 & 3200,43 & 0,0001 & 20,03 \\
16 & 1,2261 & $-1,9851$ & 2,9734 & $-2,2284$ & & & 0,9659 & 5759,01 & 0,0001 & 7,69 \\
17 & 1,2747 & $-3,4690$ & 10,7589 & $-15,4391$ & 7,0134 & & 0,9758 & 6131,08 & 0,0001 & 5,98 \\
18 & 1,2990 & $-4,5621$ & 19,8948 & $-42,3827$ & 39,4540 & $-13,6577$ & 0,9770 & 2058,63 & 0,0001 & 5,89 \\
\hline
\end{tabular}

Sendo: $b_{0}, b_{1}, b_{2}, b_{3}, b_{4}$ e $b_{5}=$ coeficientes; $R_{\text {ajus }}^{2}=$ coeficiente de determinação ajustado; $S_{y x}=$ erro padrão da estimativa; $\mathrm{F}=$ valor de $\mathrm{F}$ da análise da variância; $\mathrm{Prob}>\mathrm{F}=$ probabilidade do valor de $\mathrm{F}$ tabelado ser maior que o $\mathrm{F}$ calculado; $\mathrm{CV}=$ coeficiente de variação em percentagem.

O polinômio de $5^{\circ}$ grau, modelo 18 (Tabela 1), foi também utilizado para descrever o comprimento das toras, invertendo as variáveis dependentes e independentes, passando-se, nesse caso, a variável dependente $\left(h_{i} / h\right)$ e variáveis independentes $\left(d_{i} / d\right)$. Esse modelo apresentou um coeficiente de determinação de 0,98 , um erro padrão de estimativa de 0,05 e um coeficiente de variação igual a 5,89\%, indicando alta precisão estatística. Esse polinômio apresentou como coeficientes $b_{0}=1,2990, b_{1}=-4,5621, b_{2}=19,8948, b_{3}=-42,3827, b_{4}=39,4540$ e $b_{5}=-13,6577$. 
A mesma equação foi utilizada por SCHNEIDER (1986) para sortimentos de Pinus elliottii, da Floresta Nacional de Passo Fundo - RS, tendo encontrado um $\mathrm{R}^{2}$ de 0,96 e um $\mathrm{S}_{\mathrm{yx}}$ de 0,0579, valores semelhantes aos obtidos neste trabalho.

$\mathrm{O}$ ajuste do modelo, aos dados de altura sobre o diâmetro, resultou nos coeficientes $\mathrm{b}_{0}$ e $\mathrm{b}_{1}$ de 0,91372 e $-0,1082$, respectivamente. Essa equação apresentou um $\mathrm{R}_{\text {ajus }}$ igual a 0,98 e o $\mathrm{CV}$ de $7,32 \%$, bem como distribuição uniforme e sem tendências dos resíduos, podendo estimar a relação hipsométrica dos dados amostrados com precisão.

As tabelas 3 e 4 foram gerados para amplitudes de diâmetro de 14 a $35 \mathrm{~cm}$ ao nível do dap, com variação $\mathrm{h} / \mathrm{d}$ entre 0,56 e 0,96 na menor classe diamétrica e 0,34 e 0,74 na maior.

Nessas tabelas de sortimentos são apresentados os d (diâmetro à altura do peito, em cm), $\mathrm{h}$ (altura total, em m), h14 (altura com diâmetro de $14 \mathrm{~cm}$ com casca, em m), h/d (relação da altura total sobre o diâmetro à altura do peito), $v 7$ (volume até a altura com diâmetro com casca de $7 \mathrm{~cm}$, $\mathrm{em} \mathrm{m}^{3}$ ), v14 (volume até a altura com diâmetro com casca de $14 \mathrm{~cm}$, em $\mathrm{m}^{3}$ ), \% (percentagem de volume obtido até $14 \mathrm{~cm}$ de diâmetro em relação ao volume total), v7-14 ( volume da tora com casca com diâmetro entre 7 e $14 \mathrm{~cm}$ ), e os sortimentos de $v 14$ para as classes S1, S2 e S3, expressos em número de toras (n) e em percentagem do volume com casca, com diâmetro até $14 \mathrm{~cm}$.

Os sortimentos, apresentados na Tabela 3, foram formados, priorizando as classes de maiores dimensões de diâmetro e comprimento, sendo o volume do fuste restante aproveitado numa das classes de sortimentos com menores dimensões.

$\mathrm{Na}$ Tabela 4, também se encontram os sortimentos para as classes S1, S2 e S3, porém agrupados para uma única dimensão de tora, ou seja, apresenta o número de toras e a percentagem de volume para cada classe de sortimentos separadamente, como se as demais não existissem.

Assim, pode-se obter, na Tabela 3, para cada árvore, o número de toras das três classes de sortimentos estudadas simultaneamente, priorizando-se as de maior dimensões. Já na Tabela 4 obtém-se, para cada árvore, o número de toras por cada classe de sortimentos isoladamente, facilitando a quantificação da árvore em toras com dimensões desejadas.

\section{CONCLUSÕES}

A função polinomial de $5^{\circ}$ grau demonstra ser muito eficiente na estimativa dos diâmetros relativos ao longo do tronco e a sua integração possibilita o cálculo preciso do volume individual da árvore. Apresenta coeficiente de determinação igual a 0,98 e coeficiente de variação de $6,50 \%$, portanto, uma alta precisão e muito bom ajuste.

O polinômio de $5^{\circ}$ grau mostra grande flexibilidade, facilidade de determinação dos seus coeficientes e de integração, permitindo calcular o volume de qualquer parte do tronco. $\mathrm{O}$ volume derivado dessa equação apresenta vantagem em estimar de maneira satisfatória e com boa precisão o volume total e volumes parciais, além de possibilitar a estimativa do diâmetro em diversas alturas relativas e também da altura em diversos diâmetros relativos.

Ciência Florestal, v. 9, n. 2, 1999 


\section{REFERÊNCIAS BIBLIOGRÁFICAS}

AHRENS, S. Análise de componentes principais e a simulação da forma do tronco. In: SEMINÁRIO SOBRE ATUALIDADES E PERSPECTIVAS FLORESTAIS: o uso de funções de forma de tronco em estudos de volumetria de espécies florestais, 5; 1982, Curitiba. Anais ... Curitiba: EMBRAPA, URPFCS, 1982. p. 77-92.

BENNETT, F.A.; SWINDEL, B.F. Taper curves for planted slash pine. USDA Forest Service (RN SE-179), 1972. 4p.

BORGES, J.F. Seccionamento do fuste de Pinus taeda L. para obtenção do volume de madeira serrada através da função polinomial. Curitiba: UFPR, 1981. 81 p. Dissertação (Mestrado em Ciências Florestais). Setor de Ciências Agrárias, Universidade Federal do Paraná, 1981.

FIGUEREDO-FILHO, A., BORDERS, B.E., HITCH, K.L. Taper equations for Pinus taeda L. plantations in Southern Brazil. Forest Ecology and Management, v.83, 1996. p. 39-46.

FINGER, C.A.G. Fundamentos de biometria florestal. Santa Maria: UFSM, CEPEF - FATEC, 1992. $269 \mathrm{p}$.

FINGER, C.A.G., ZANON, M.L.B., SCHNEIDER, P.R. et al. Funções de forma para Eucalyptus dunnii Maiden implantados na depressão central e encosta do sudeste do Rio Grande do Sul. Santa Maria - RS. Ciência Rural, v.25, n.3, p. 399-403, 1995.

FRIEDL, R.A. Dinâmica e prognose da forma dos fustes em povoamentos plantados de Araucaria angustifolia (Bert.) O. Ktze. Curitiba: UFPR, 1989. 167p. Dissertação (Mestrado em Ciências Florestais) - Setor de Ciências Agrárias, Universidade Federal do Paraná, 1989.

GRAY, H.R. The form and taper of forest-tree stems. Imp.For.Inst. Paper, Oxford, v.42. 1956. p. $1-75$.

JORGE, L.A.B. Tabelas de sortimento para Pinus elliottii Engelm, na Floresta Nacional de Três Barras - SC. Revista Floresta, Curitiba. v. 15, n.1/2, p.12-20, 1984.

JORGE, L.A.B.; LARA, H.A. Programa de sortimento de madeira serrada de povoamentos de Pinus elliotii com alternativas de produtos padronizados. In: CONGRESSO FLORESTAL PANAMERICANO, 1. / CONGRESSO FLORESTAL BRASILEIRO, 7., 1993, Curitiba. Anais...Curitiba:SBS/SBEF,1993. P.539-548

KOEHLER, H.S. Funções de forma em inventários florestais: implementação e processamento do sistema. In: SEMINÁRIO SOBRE ATUALIDADES E PERSPECTIVAS FLORESTAIS: o uso de funções de forma de tronco em estudos de volumetria de espécies florestais, 5., 1982, Curitiba. Anais ... Curitiba: EMBRAPA, URPFCS, 1982. p. 93-96.

KOZAK, A., MUNRO, D.D.; SMITH, J.H.G. Taper Functions and their Applications in Forest Inventory. For. Chron., v. 45, n. 4, 1969. p.278-283.

LEMOS, R.C., AZOLIN, M.A.D., ABRAÃO, P.V.R., et al. Levantamento e reconhecimento dos solos do Estado do Rio Grande do Sul. Recife: Ministério da Agricultura, Departamento 
Nacional de Pesquisas Agropecuárias - Divisão de Pesquisas Pedológicas, 1973. 431 p. (Boletim Técnico, 30).

MAINARDI, G.L. Estruturação da produção de Pinus taeda L.: um estudo do caso para a região de Cambará do Sul, RS. Santa Maria: UFSM, 1995. 77 p. Dissertação (Mestrado em Engenharia Florestal) - Universidade Federal de Santa Maria, 1995.

MORENO, J.A. Clima do Rio Grande do Sul. Porto Alegre: Secretaria da Agricultura, 1961. 42p.

MOURA, J.B. de Estudo da forma do fuste e comparação de métodos de estimativas volumétricas de espécies florestais da Amazônia Brasileira. Curitiba: UFPR, 1994. 114 p. Dissertação (Mestrado em Ciências Florestais) - Setor de Ciências Agrárias, Universidade Federal do Paraná, 1994.

MUNRO, D.D. ; DEMAERSCHALK, J. Taper-based versus volume based compatible estimating systems. For. Chron., v.50, no5, 1974. p.197-199.

SCHNEIDER, P.R. Betriebswirtschaftliche und ertragskundliche Grundlagen der Forteinnrichtung in Südbrasilien am Beispiel von Pinus elliottii. Diss. d. Albert-LudwigsUniversitat Freiburg, 1984. 190p.

- Forma de tronco e sortimento para Pinus elliottii Engelm., da floresta nacional de Passo Fundo, Rio Grande do Sul. Acta For. Bras., Curitiba, v.1, p.43-64. 1986.

— Introdução ao manejo florestal. Santa Maria - RS: UFSM/ CEPEF-FATEC, 1993. 348 p.

SCHNEIDER, P.R., FINGER, C.A.G., KLEIN, J.E.M., et al. Forma de tronco e sortimentos de madeira de Eucalyptus grandis Maiden. para o Estado do Rio Grande do Sul. Ciência Florestal, Santa Maria, v.7, n.1, p. 85-91. 1996.

SILVA, J.A. Seleção de parcelas amostrais aplicadas em povoamentos de Pinus taeda L. para fins biométricos em Santa Maria - RS. Santa Maria: UFSM, 1974. 87p. Dissertação (Mestrado em Engenharia Rural) - Universidade Federal de Santa Maria, 1974.

- Funções de forma dos troncos de Pinus taeda, Picea excelsa, Abies alba e Pinus sylvestris. In: SEMINÁRIO SOBRE ATUALIDADES E PERSPECTIVAS FLORESTAIS: o uso de funções de forma de tronco em estudos de volumetria de espécies florestais, 5; 1982, Curitiba. Anais ... Curitiba: EMBRAPA, URPFCS, 1982. p. $29-45$.

- Funções de forma dos troncos de Pinus taeda, Picea excelsa, Abies alba e Pinus sylvestris. In: SEMINÁRIO SOBRE ATUALIDADES E PERSPECTIVAS FLORESTAIS: o uso de funções de forma de tronco em estudos de volumetria de espécies florestais, 5; 1982, Curitiba. Anais ... Curitiba: EMBRAPA, URPFCS, 1983. p. 29 - 45.

SAS. Statistical analysis system: programa de computador, ambiente VM. Cary, 1993. Versão 6.08 . 
TABELA 3: Tabela de sortimento otimizada para árvores individuais de Pinus elliottii para o diâmetro mínimo na ponta fina da tora igual a $14 \mathrm{~cm}$.

\begin{tabular}{|c|c|c|c|c|c|c|c|c|c|c|c|c|c|}
\hline \multirow{3}{*}{$\begin{array}{l}\text { DAP } \\
\mathrm{cm}\end{array}$} & \multirow{3}{*}{$\begin{array}{l}\mathrm{H} \\
\mathrm{M}\end{array}$} & \multirow{3}{*}{$\begin{array}{c}\mathrm{h} 14 \\
\mathrm{~m}\end{array}$} & \multirow[t]{3}{*}{$\mathrm{h} / \mathrm{d}$} & \multirow{3}{*}{$\begin{array}{c}\mathrm{v} 7 \\
\mathrm{~m}^{3} \mathrm{c} / \mathrm{c}\end{array}$} & \multirow{3}{*}{$\begin{array}{c}\mathrm{v} 14 \\
\mathrm{~m}^{3} \mathrm{c} / \mathrm{c}\end{array}$} & \multirow{3}{*}{$\begin{array}{l}\mathrm{v} 14 \\
\% \\
\end{array}$} & \multirow{3}{*}{$\begin{array}{l}\mathrm{v} 7-14 \\
\mathrm{~m}^{3} \mathrm{c} / \mathrm{c}\end{array}$} & \multicolumn{6}{|c|}{ Sortimento (v14) } \\
\hline & & & & & & & & \multicolumn{2}{|c|}{ S1 } & \multicolumn{2}{|c|}{ S2 } & \multicolumn{2}{|c|}{ S3 } \\
\hline & & & & & & & & $\mathrm{n}$ & $\%$ & $\mathrm{n}$ & $\%$ & $\mathrm{n}$ & $\%$ \\
\hline 14,0 & 7,9 & 0,9 & 0,56 & 0,0599 & 0,0165 & 27,5 & 0,0435 & 0 & 0,0 & 0 & 0,0 & 0 & 0,0 \\
\hline 14,0 & 9,3 & 1,0 & 0,66 & 0,0706 & 0,0194 & 27,5 & 0,0512 & 0 & 0,0 & 0 & 0,0 & 0 & 0,0 \\
\hline 14,0 & 10,7 & 1,2 & 0,76 & 0,0812 & 0,0223 & 27,5 & 0,0589 & 0 & 0,0 & 0 & 0,0 & 0 & 0,0 \\
\hline 14,0 & 12,1 & 1,3 & 0,86 & 0,0919 & 0,0253 & 27,5 & 0,0666 & 0 & 0,0 & 0 & 0,0 & 1 & 99,6 \\
\hline 14,0 & 13,5 & 1,5 & 0,96 & 0,1026 & 0,0282 & 27,5 & 0,0744 & 0 & 0,0 & 0 & 0,0 & 1 & 91,4 \\
\hline 15,0 & 8,3 & 1,6 & 0,55 & 0,0728 & 0,0308 & 42,2 & 0,0420 & 0 & 0,0 & 0 & 0,0 & 1 & 85,9 \\
\hline 15,0 & 9,8 & 1,9 & 0,65 & 0,0860 & 0,0363 & 42,3 & 0,0497 & 0 & 0,0 & 0 & 0,0 & 1 & 75,7 \\
\hline 15,0 & 11,3 & 2,2 & 0,75 & 0,0992 & 0,0419 & 42,3 & 0,0573 & 0 & 0,0 & 1 & 94,0 & 0 & 0,0 \\
\hline 15,0 & 12,8 & 2,5 & 0,85 & 0,1124 & 0,0475 & 42,2 & 0,0649 & 0 & 0,0 & 1 & 85,6 & 0 & 0,0 \\
\hline 15,0 & 14,3 & 2,7 & 0,95 & 0,1256 & 0,0531 & 42,2 & 0,0725 & 0 & 0,0 & 1 & 78,8 & 0 & 0,0 \\
\hline 16,0 & 8,6 & 2,4 & 0,54 & 0,0870 & 0,0476 & 54,8 & 0,0394 & 0 & 0,0 & 1 & 88,0 & 0 & 0,0 \\
\hline 16,0 & 10,2 & 2,8 & 0,64 & 0,1031 & 0,0565 & 54,8 & 0,0467 & 1 & 99,7 & 0 & 0,0 & 0 & 0,0 \\
\hline 16,0 & 11,8 & 3,2 & 0,74 & 0,1192 & 0,0653 & 54,8 & 0,0539 & 1 & 89,5 & 0 & 0,0 & 0 & 0,0 \\
\hline 16,0 & 13,4 & 3,7 & 0,84 & 0,1353 & 0,0741 & 54,8 & 0,0612 & 1 & 81,4 & 0 & 0,0 & 0 & 0,0 \\
\hline 16,0 & 15,0 & 4,1 & 0,94 & 0,1514 & 0,0829 & 54,8 & 0,0685 & 1 & 74,8 & 0 & 0,0 & 1 & 24,7 \\
\hline 17,0 & 9,0 & 3,1 & 0,53 & 0,1026 & 0,0670 & 65,3 & 0,0356 & 1 & 91,8 & 0 & 0,0 & 0 & 0,0 \\
\hline 17,0 & 10,7 & 3,7 & 0,63 & 0,1220 & 0,0797 & 65,3 & 0,0423 & 1 & 80,7 & 0 & 0,0 & 0 & 0,0 \\
\hline 17,0 & 12,4 & 4,3 & 0,73 & 0,1414 & 0,0923 & 65,3 & 0,0490 & 1 & 72,2 & 0 & 0,0 & 1 & 23,8 \\
\hline 17,0 & 14,1 & 4,9 & 0,83 & 0,1607 & 0,1050 & 65,3 & 0,0558 & 1 & 65,6 & 1 & 32,5 & 0 & 0,0 \\
\hline 17,0 & 15,8 & 5,5 & 0,93 & 0,1801 & 0,1176 & 65,3 & 0,0625 & 1 & 60,3 & 1 & 29,9 & 0 & 0,0 \\
\hline 18,0 & 9,3 & 3,9 & 0,52 & 0,1196 & 0,0883 & 73,8 & 0,0313 & 1 & 78,8 & 0 & 0,0 & 0 & 0,0 \\
\hline 18,0 & 11,1 & 4,6 & 0,62 & 0,1426 & 0,1053 & 73,8 & 0,0373 & 1 & 69,1 & 0 & 0,0 & 1 & 22,7 \\
\hline 18,0 & 12,9 & 5,4 & 0,72 & 0,1657 & 0,1223 & 73,8 & 0,0433 & 1 & 61,8 & 1 & 30,5 & 0 & 0,0 \\
\hline 18,0 & 14,7 & 6,1 & 0,82 & 0,1887 & 0,1393 & 73,8 & 0,0494 & 2 & 93,9 & 0 & 0,0 & 0 & 0,0 \\
\hline 18,0 & 16,5 & 6,9 & 0,92 & 0,2118 & 0,1564 & 73,8 & 0,0554 & 2 & 86,3 & 0 & 0,0 & 0 & 0,0 \\
\hline 19,0 & 9,7 & 4,6 & 0,51 & 0,1380 & 0,1109 & 80,4 & 0,0270 & 1 & 70,5 & 0 & 0,0 & 1 & 22,6 \\
\hline
\end{tabular}

Continua ... 
TABELA 3: Continuação ...

\begin{tabular}{|c|c|c|c|c|c|c|c|c|c|c|c|c|c|}
\hline \multirow{3}{*}{\begin{tabular}{c|} 
DAP \\
$\mathrm{cm}$ \\
\end{tabular}} & \multirow{3}{*}{$\begin{array}{l}\mathrm{H} \\
\mathrm{M}\end{array}$} & \multirow{3}{*}{$\begin{array}{c}\mathrm{h} 14 \\
\mathrm{~m}\end{array}$} & \multirow[t]{3}{*}{$\mathrm{h} / \mathrm{d}$} & \multirow{3}{*}{$\begin{array}{c}\mathrm{v} 7 \\
\mathrm{~m}^{3} \mathrm{c} / \mathrm{c}\end{array}$} & \multirow{3}{*}{$\begin{array}{c}\mathrm{v} 14 \\
\mathrm{~m}^{3} \mathrm{c} / \mathrm{c}\end{array}$} & \multirow{3}{*}{$\begin{array}{l}\mathrm{v} 14 \\
\%\end{array}$} & \multirow{3}{*}{$\begin{array}{l}\mathrm{v} 7-14 \\
\mathrm{~m}^{3} \mathrm{c} / \mathrm{c}\end{array}$} & \multicolumn{6}{|c|}{ Sortimento (v14) } \\
\hline & & & & & & & & \multicolumn{2}{|c|}{ S1 } & \multicolumn{2}{|c|}{$\mathrm{S} 2$} & \multicolumn{2}{|c|}{ S3 } \\
\hline & & & & & & & & $\mathrm{n}$ & $\%$ & $\bar{n}$ & $\%$ & $\mathrm{n}$ & $\%$ \\
\hline 19,0 & 11,6 & 5,5 & 0,61 & 0,1651 & 0,1328 & 80,4 & 0,0324 & 1 & 61,6 & 1 & 30,1 & 0 & 0,0 \\
\hline 19,0 & 13,5 & 6,4 & 0,71 & 0,1922 & 0,1546 & 80,4 & 0,0377 & 2 & 91,9 & 0 & 0,0 & 0 & 0,0 \\
\hline 19,0 & 15,4 & 7,3 & 0,81 & 0,2194 & 0,1764 & 80,4 & 0,0430 & 2 & 83,6 & 0 & 0,0 & 1 & 13,2 \\
\hline 19,0 & 17,3 & 8,2 & 0,91 & 0,2466 & 0,1982 & 80,4 & 0,0483 & 2 & 76,7 & 1 & 18,8 & 0 & 0,0 \\
\hline 20,0 & 9,9 & 5,2 & 0,50 & 0,1577 & 0,1345 & 85,3 & 0,0232 & 1 & 64,9 & 1 & 30,5 & 0 & 0,0 \\
\hline 20,0 & 11,9 & 6,2 & 0,60 & 0,1894 & 0,1615 & 85,3 & 0,0279 & 2 & 93,7 & 0 & 0,0 & 0 & 0,0 \\
\hline 20,0 & 13,9 & 7,3 & 0,70 & 0,2211 & 0,1886 & 85,3 & 0,0325 & 2 & 84,3 & 0 & 0,0 & 1 & 12,5 \\
\hline 20,0 & 15,9 & 8,3 & 0,80 & 0,2528 & 0,2156 & 85,3 & 0,0372 & 2 & 76,5 & 1 & 18,1 & 0 & 0,0 \\
\hline 20,0 & 17,9 & 9,4 & 0,90 & 0,2845 & 0,2427 & 85,3 & 0,0419 & 3 & 93,6 & 0 & 0,0 & 0 & 0,0 \\
\hline 21,0 & 10,2 & 5,8 & 0,49 & 0,1788 & 0,1588 & 88,8 & 0,0199 & 2 & 98,5 & 0 & 0,0 & 0 & 0,0 \\
\hline 21,0 & 12,3 & 7,0 & 0,59 & 0,2155 & 0,1915 & 88,8 & 0,0240 & 2 & 88,0 & 0 & 0,0 & 1 & 11,6 \\
\hline 21,0 & 14,4 & 8,1 & 0,69 & 0,2523 & 0,2241 & 88,8 & 0,0281 & 2 & 79,0 & 1 & 17,4 & 0 & 0,0 \\
\hline 21,0 & 16,5 & 9,3 & 0,79 & 0,2890 & 0,2568 & 88,8 & 0,0322 & 3 & 94,4 & 0 & 0,0 & 0 & 0,0 \\
\hline 21,0 & 18,6 & 10,5 & 0,89 & 0,3257 & 0,2894 & 88,9 & 0,0363 & 3 & 87,6 & 1 & 11,9 & 0 & 0,0 \\
\hline 22,0 & 10,5 & 6,3 & 0,48 & 0,2012 & 0,1839 & 91,4 & 0,0172 & 2 & 94,4 & 0 & 0,0 & 0 & 0,0 \\
\hline 22,0 & 12,7 & 7,6 & 0,58 & 0,2435 & 0,2226 & 91,4 & 0,0209 & 2 & 83,9 & 1 & 16,1 & 0 & 0,0 \\
\hline 22,0 & 14,9 & 8,9 & 0,68 & 0,2858 & 0,2613 & 91,4 & 0,0245 & 3 & 97,1 & 0 & 0,0 & 0 & 0,0 \\
\hline 22,0 & 17,1 & 10,2 & 0,78 & 0,3281 & 0,2999 & 91,4 & 0,0281 & 3 & 89,9 & 0 & 0,0 & 1 & 7,5 \\
\hline 22,0 & 19,3 & 11,6 & 0,88 & 0,3704 & 0,3386 & 91,4 & 0,0318 & 4 & 98,5 & 0 & 0,0 & 0 & 0,0 \\
\hline 23,0 & 10,7 & 6,7 & 0,46 & 0,2248 & 0,2098 & 93,3 & 0,0151 & 2 & 91,3 & 0 & 0,0 & 0 & $\overline{0,0}$ \\
\hline 23,0 & 13,0 & 8,2 & 0,56 & 0,2732 & 0,2549 & 93,3 & 0,0183 & 2 & 80,7 & 1 & 16,0 & 0 & 0,0 \\
\hline 23,0 & 15,3 & 9,7 & 0,66 & 0,3216 & 0,3000 & 93,3 & 0,0215 & 3 & 93,7 & 0 & 0,0 & 0 & 0,0 \\
\hline 23,0 & 17,6 & 11,1 & 0,76 & 0,3699 & 0,3451 & 93,3 & 0,0248 & 3 & 86,4 & 1 & 10,8 & 0 & 0,0 \\
\hline 23,0 & 19,9 & 12,6 & 0,86 & 0,4183 & 0,3903 & 93,3 & 0,0281 & 4 & 94,9 & 0 & 0,0 & 1 & 4,9 \\
\hline 24,0 & 10,9 & 7,2 & 0,45 & 0,2497 & 0,2364 & 94,7 & 0,0133 & 2 & 88,9 & 0 & 0,0 & 1 & 9,7 \\
\hline 24,0 & 13,3 & 8,7 & 0,55 & 0,3047 & 0,2884 & 94,7 & 0,0162 & 3 & 98,5 & 0 & 0,0 & 0 & 0,0 \\
\hline 24,0 & 15,7 & 10,3 & 0,65 & 0,3597 & 0,3405 & 94,7 & 0,0192 & 3 & 91,0 & 0 & 0,0 & 1 & 6,6 \\
\hline 24,0 & 18,1 & 11,9 & 0,75 & 0,4147 & 0,3925 & 94,7 & 0,0221 & 4 & 97,7 & 0 & 0,0 & 0 & 0,0 \\
\hline 24,0 & 20,5 & 13,5 & 0,85 & 0,4697 & 0,4446 & 94,7 & 0,0251 & 4 & 92,0 & 1 & 7,2 & 0 & 0,0 \\
\hline
\end{tabular}


TABELA 3: Continuação ...

\begin{tabular}{|c|c|c|c|c|c|c|c|c|c|c|c|c|c|}
\hline \multirow{3}{*}{$\begin{array}{l}\mathrm{DAP} \\
\mathrm{Cm}\end{array}$} & \multirow{3}{*}{$\begin{array}{l}\mathrm{H} \\
\mathrm{M}\end{array}$} & \multirow{3}{*}{$\begin{array}{c}\mathrm{h} 14 \\
\mathrm{~m}\end{array}$} & \multirow[t]{3}{*}{$h / d$} & \multirow{3}{*}{$\begin{array}{c}\mathrm{v} 7 \\
\mathrm{~m}^{3} \mathrm{c} / \mathrm{c} \\
\end{array}$} & \multirow{3}{*}{$\begin{array}{c}\mathrm{v} 14 \\
\mathrm{~m}^{3} \mathrm{c} / \mathrm{c}\end{array}$} & \multirow{3}{*}{$\begin{array}{l}\text { v14 } \\
\%\end{array}$} & \multirow{3}{*}{$\begin{array}{l}\mathrm{v} 7-14 \\
\mathrm{~m}^{3} \mathrm{c} / \mathrm{c}\end{array}$} & \multicolumn{6}{|c|}{ Sortimento (v14) } \\
\hline & & & & & & & & \multicolumn{2}{|c|}{ S1 } & \multicolumn{2}{|c|}{ S2 } & \multicolumn{2}{|c|}{ S3 } \\
\hline & & & & & & & & $\mathrm{n}$ & $\%$ & $\mathrm{n}$ & $\%$ & $\mathrm{n}$ & $\%$ \\
\hline 25,0 & 11,1 & $\overline{7,5}$ & 0,44 & 0,2757 & 0,2638 & 95,7 & 0,0119 & $\overline{2}$ & 87,1 & $\overline{0}$ & $\overline{0,0}$ & 1 & 9,8 \\
\hline 25,0 & 13,6 & 9,2 & 0,54 & 0,3379 & 0,3233 & 95,7 & 0,0146 & 3 & 96,6 & 0 & 0,0 & 0 & 0,0 \\
\hline 25,0 & 16,1 & 10,9 & 0,64 & 0,4001 & 0,3828 & 95,7 & 0,0173 & 3 & 88,8 & 1 & 9,5 & 0 & 0,0 \\
\hline 25,0 & 18,6 & 12,6 & 0,74 & 0,4623 & 0,4423 & 95,7 & 0,0201 & 4 & 95,5 & 0 & 0,0 & 1 & 4,2 \\
\hline 25,0 & 21,1 & 14,3 & 0,84 & 0,5245 & 0,5018 & 95,7 & 0,0227 & 5 & 99,2 & 0 & 0,0 & 0 & 0,0 \\
\hline 26,0 & 11,2 & 7,9 & 0,43 & 0,3028 & 0,2919 & 96,4 & 0,0109 & 2 & 85,6 & 1 & 13,4 & 0 & 0,0 \\
\hline 26,0 & 13,8 & 9,7 & 0,53 & 0,3728 & 0,3594 & 96,4 & 0,0134 & 3 & 95,0 & 0 & 0,0 & 0 & 0,0 \\
\hline 26,0 & 16,4 & 11,5 & 0,63 & 0,4428 & 0,4269 & 96,4 & 0,0159 & 4 & 99,2 & 0 & 0,0 & 0 & 0,0 \\
\hline 26,0 & 19,0 & 13,3 & 0,73 & 0,5128 & 0,4944 & 96,4 & 0,0184 & 4 & 93,6 & 1 & 6,1 & 0 & 0,0 \\
\hline 26,0 & 21,6 & 15,1 & 0,83 & 0,5828 & 0,5619 & 96,4 & 0,0209 & 5 & 97,5 & 0 & 0,0 & 0 & 0,0 \\
\hline 27,0 & 11,4 & 8,1 & 0,42 & 0,3307 & 0,3208 & 97,0 & 0,0100 & 2 & 84,4 & 1 & 13,5 & 0 & 0,0 \\
\hline 27,0 & 14,1 & 10,1 & 0,52 & 0,4092 & 0,3968 & 97,0 & 0,0123 & 3 & 93,7 & 0 & 0,0 & 1 & 5,3 \\
\hline 27,0 & 16,8 & 12,0 & 0,62 & 0,4876 & 0,4729 & 97,0 & 0,0147 & 4 & 97,9 & 0 & 0,0 & 0 & 0,0 \\
\hline 27,0 & 19,5 & 13,9 & 0,72 & 0,5661 & 0,5490 & 97,0 & 0,0171 & 4 & 92,0 & 1 & 6,4 & 0 & 0,0 \\
\hline 27,0 & 22,2 & 15,9 & 0,82 & 0,6445 & 0,6251 & 97,0 & 0,0194 & 5 & 95,9 & 0 & 0,0 & 1 & 3,0 \\
\hline 28,0 & 11,5 & 8,4 & 0,41 & 0,3596 & 0,3503 & 97,4 & 0,0093 & 2 & 83,5 & 1 & 13,6 & 0 & 0,0 \\
\hline 28,0 & 14,3 & 10,4 & 0,51 & 0,4471 & 0,4356 & 97,4 & 0,0115 & 3 & 92,5 & 1 & 7,4 & 0 & 0,0 \\
\hline 28,0 & 17,1 & 12,5 & 0,61 & 0,5346 & 0,5208 & 97,4 & 0,0138 & 4 & 96,8 & 0 & 0,0 & 0 & 0,0 \\
\hline 28,0 & 19,9 & 14,5 & 0,71 & 0,6222 & 0,6061 & 97,4 & 0,0161 & 5 & 99,1 & 0 & 0,0 & 0 & 0,0 \\
\hline 28,0 & 22,7 & 16,5 & 0,81 & 0,7097 & 0,6914 & 97,4 & 0,0184 & 5 & 94,6 & 1 & 4,6 & 0 & 0,0 \\
\hline 29,0 & 11,6 & 8,6 & 0,40 & 0,3892 & 0,3804 & 97,7 & 0,0088 & 3 & 99,5 & 0 & 0,0 & 0 & 0,0 \\
\hline 29,0 & 14,5 & 10,7 & 0,50 & 0,4865 & 0,4755 & 97,7 & 0,0110 & 3 & 91,6 & 1 & 7,6 & 0 & 0,0 \\
\hline 29,0 & 17,4 & 12,9 & 0,60 & 0,5837 & 0,5706 & 97,8 & 0,0131 & 4 & 95,8 & 0 & 0,0 & 1 & 3,4 \\
\hline 29,0 & 20,3 & 15,0 & 0,70 & 0,6811 & 0,6657 & 97,7 & 0,0154 & 5 & 98,1 & 0 & 0,0 & 0 & 0,0 \\
\hline 29,0 & 23,2 & 17,2 & 0,80 & 0,7784 & 0,7608 & 97,7 & 0,0176 & 6 & 99,5 & 0 & 0,0 & 0 & 0,0 \\
\hline 30,0 & 11,7 & 8,8 & 0,39 & 0,4194 & 0,4110 & 98,0 & 0,0084 & 3 & 99,0 & 0 & 0,0 & 0 & 0,0 \\
\hline 30,0 & 14,7 & 11,0 & 0,49 & 0,5271 & 0,5166 & 98,0 & 0,0105 & 3 & 90,8 & 1 & 7,8 & 0 & 0,0 \\
\hline 30,0 & 17,7 & 13,3 & 0,59 & 0,6349 & 0,6223 & 98,0 & 0,0127 & 4 & 94,9 & 1 & 4,9 & 0 & 0,0 \\
\hline 30,0 & 20,7 & 15,5 & 0,69 & 0,7427 & 0,7279 & 98,0 & 0,0148 & 5 & 97,2 & 0 & 0,0 & 1 & 2,4 \\
\hline
\end{tabular}

Continua ... 
TABELA 3: Continuação ...

\begin{tabular}{|c|c|c|c|c|c|c|c|c|c|c|c|c|c|}
\hline \multirow{3}{*}{$\begin{array}{l}\text { DAP } \\
\mathrm{Cm}\end{array}$} & \multirow{3}{*}{$\begin{array}{l}\mathrm{H} \\
\mathrm{M} \\
\end{array}$} & \multirow{3}{*}{$\begin{array}{r}\mathrm{h} 14 \\
\mathrm{~m}\end{array}$} & \multirow[t]{3}{*}{$\mathrm{h} / \mathrm{d}$} & \multirow{3}{*}{$\begin{array}{c}\mathrm{v} 7 \\
\mathrm{~m}^{3} \mathrm{c} / \mathrm{c}\end{array}$} & \multirow{3}{*}{$\begin{array}{c}\mathrm{v} 14 \\
\mathrm{~m}^{3} \mathrm{c} / \mathrm{c}\end{array}$} & \multirow{3}{*}{$\begin{array}{l}\text { v14 } \\
\%\end{array}$} & \multirow{3}{*}{$\begin{array}{l}\mathrm{v} 7-14 \\
\mathrm{~m}^{3} \mathrm{c} / \mathrm{c}\end{array}$} & \multicolumn{6}{|c|}{ Sortimento (v14) } \\
\hline & & & & & & & & \multicolumn{2}{|c|}{ S1 } & \multicolumn{2}{|c|}{ S2 } & \multicolumn{2}{|c|}{ S3 } \\
\hline & & & & & & & & $\mathrm{n}$ & $\%$ & $\mathrm{n}$ & $\%$ & $\mathrm{n}$ & $\%$ \\
\hline 30,0 & 23,7 & 17,8 & 0,79 & 0,8504 & 0,8335 & 98,0 & 0,0169 & 6 & 98,6 & 0 & 0,0 & 0 & 0,0 \\
\hline 31,0 & 11,7 & 8,9 & 0,38 & 0,4500 & 0,4420 & 98,2 & 0,0080 & 3 & 98,6 & 0 & 0,0 & 0 & 0,0 \\
\hline 31,0 & 14,8 & 11,3 & 0,48 & 0,5690 & 0,5588 & 98,2 & 0,0102 & 4 & 99,9 & 0 & 0,0 & 0 & 0,0 \\
\hline 31,0 & 17,9 & 13,6 & 0,58 & 0,6879 & 0,6757 & 98,2 & 0,0123 & 4 & 94,2 & 1 & 5,1 & 0 & 0,0 \\
\hline 31,0 & 21,0 & 16,0 & 0,68 & 0,8069 & 0,7925 & 98,2 & 0,0144 & 5 & 96,5 & 0 & 0,0 & 1 & 2,6 \\
\hline 31,0 & 24,1 & 18,3 & 0,78 & 0,9258 & 0,9093 & 98,2 & 0,0165 & 6 & 97,8 & 0 & 0,0 & 1 & 1,9 \\
\hline 32,0 & 11,8 & 9,0 & 0,37 & 0,4810 & 0,4732 & 98,4 & 0,0078 & 3 & 98,3 & 0 & 0,0 & 0 & 0,0 \\
\hline 32,0 & 15,0 & 11,5 & 0,47 & 0,6120 & 0,6020 & 98,4 & 0,0099 & 4 & 99,5 & 0 & 0,0 & 0 & 0,0 \\
\hline 32,0 & 18,2 & 13,9 & 0,57 & 0,7428 & 0,7308 & 98,4 & 0,0121 & 4 & 93,5 & 1 & 5,3 & 0 & 0,0 \\
\hline 32,0 & 21,4 & 16,4 & 0,67 & 0,8737 & 0,8596 & 98,4 & 0,0141 & 5 & 95,7 & 1 & 3,8 & 0 & 0,0 \\
\hline 32,0 & 24,6 & 18,9 & 0,77 & 1,0046 & 0,9883 & 98,4 & 0,0163 & 6 & 97,1 & 1 & 2,8 & 0 & 0,0 \\
\hline 33,0 & 11,8 & 9,1 & 0,36 & 0,5122 & 0,5046 & 98,5 & 0,0076 & 3 & 98,2 & 0 & 0,0 & 0 & 0,0 \\
\hline 33,0 & 15,1 & 11,7 & 0,46 & 0,6559 & 0,6460 & 98,5 & 0,0098 & 4 & 99,2 & 0 & 0,0 & 0 & 0,0 \\
\hline 33,0 & 18,4 & 14,2 & 0,56 & 0,7994 & 0,7875 & 98,5 & 0,0118 & 5 & 99,7 & 0 & 0,0 & 0 & 0,0 \\
\hline 33,0 & 21,7 & 16,8 & 0,66 & 0,9431 & 0,9290 & 98,5 & 0,0141 & 5 & 95,1 & 1 & 3,9 & 0 & 0,0 \\
\hline 33,0 & 25,0 & 19,4 & 0,76 & 1,0865 & 1,0705 & 98,5 & 0,0161 & 6 & 96,4 & 1 & 3,0 & 0 & 0,0 \\
\hline 34,0 & 11,8 & 9,2 & 0,35 & 0,5434 & 0,5359 & 98,6 & 0,0074 & 3 & 98,0 & 0 & 0,0 & 0 & 0,0 \\
\hline 34,0 & 15,2 & 11,8 & 0,45 & 0,7005 & 0,6908 & 98,6 & 0,0097 & 4 & 98,9 & 0 & 0,0 & 0 & 0,0 \\
\hline 34,0 & 18,6 & 14,5 & 0,55 & 0,8577 & 0,8458 & 98,6 & 0,0119 & 5 & 99,3 & 0 & 0,0 & 0 & 0,0 \\
\hline 34,0 & 22,0 & 17,2 & 0,65 & 1,0147 & 1,0007 & 98,6 & 0,0139 & 6 & 99,6 & 0 & 0,0 & 0 & 0,0 \\
\hline 34,0 & 25,4 & 19,8 & 0,75 & 1,1718 & 1,1557 & 98,6 & 0,0161 & 7 & 99,8 & 0 & 0,0 & 0 & 0,0 \\
\hline 35,0 & 11,7 & 9,2 & 0,34 & 0,5744 & 0,5670 & 98,7 & 0,0074 & 3 & 98,0 & 0 & 0,0 & 0 & 0,0 \\
\hline 35,0 & 15,2 & 12,0 & 0,44 & 0,7459 & 0,7363 & 98,7 & 0,0096 & 4 & 98,6 & 0 & 0,0 & 0 & 0,0 \\
\hline 35,0 & 18,7 & 14,7 & 0,54 & 0,9173 & 0,9055 & 98,7 & 0,0118 & 5 & 99,0 & 0 & 0,0 & 0 & 0,0 \\
\hline 35,0 & 22,2 & 17,5 & 0,64 & 1,0887 & 1,0747 & 98,7 & 0,0140 & 6 & 99,2 & 0 & 0,0 & 0 & 0,0 \\
\hline 35,0 & 25,7 & 20,2 & 0,74 & 1,2603 & 1,2439 & 98,7 & 0,0164 & 7 & 99,4 & 0 & 0,0 & 0 & 0,0 \\
\hline
\end{tabular}

Sendo: S1 = madeira para serraria com diâmetro na ponta fina superior a $14 \mathrm{~cm}$ com casca e comprimento de 2,8 metros; S2 = madeira para serraria com diâmetro na ponta fina superior a $14 \mathrm{~cm}$ com casca e comprimento de 2,0 metros; S3 = madeira para serraria com diâmetro na ponta fina superior a $14 \mathrm{~cm}$ com casca e comprimento de 1,30 metros. 
TABELA 4: Tabela de sortimento para árvores individuais de Pinus elliottii com opções de aproveitamento em relação as dimensões das toras.

\begin{tabular}{|c|c|c|c|c|c|c|c|c|c|c|c|c|c|}
\hline \multirow{3}{*}{$\begin{array}{l}\text { DAP } \\
\mathrm{cm}\end{array}$} & \multirow{3}{*}{$\begin{array}{l}\mathrm{h} \\
\mathrm{m}\end{array}$} & \multirow{3}{*}{$\begin{array}{c}\mathrm{h} 14 \\
\mathrm{~m}\end{array}$} & \multirow[t]{3}{*}{$\mathrm{h} / \mathrm{d}$} & \multirow{3}{*}{$\begin{array}{c}\mathrm{v} 7 \\
\mathrm{~m}^{3} \mathrm{c} / \mathrm{c}\end{array}$} & \multirow{3}{*}{$\begin{array}{c}\mathrm{v} 14 \\
\mathrm{~m}^{3} \mathrm{c} / \mathrm{c}\end{array}$} & \multirow{3}{*}{$\begin{array}{c}\mathrm{v} 14 \\
\%\end{array}$} & \multirow{3}{*}{$\begin{array}{l}\mathrm{v} 7-14 \\
\mathrm{~m}^{3} \mathrm{c} / \mathrm{c}\end{array}$} & \multicolumn{6}{|c|}{ Sortimento (v14) } \\
\hline & & & & & & & & \multicolumn{2}{|c|}{ S1 } & \multicolumn{2}{|c|}{$\mathrm{S} 2$} & \multicolumn{2}{|c|}{ S3 } \\
\hline & & & & & & & & $\mathrm{n}$ & $\%$ & $\mathrm{n}$ & $\%$ & $\mathrm{n}$ & $\%$ \\
\hline 14,0 & 7,9 & 0,9 & 0,56 & 0,0599 & 0,0165 & 27,5 & 0,0435 & 0 & 0,0 & 0 & 0,0 & 0 & 0,0 \\
\hline 14,0 & 9,3 & 1,0 & 0,66 & 0,0706 & 0,0194 & 27,5 & 0,0512 & 0 & 0,0 & 0 & 0,0 & 0 & 0,0 \\
\hline 14,0 & 10,7 & 1,2 & 0,76 & 0,0812 & 0,0223 & 27,5 & 0,0589 & 0 & 0,0 & 0 & 0,0 & 0 & 0,0 \\
\hline 14,0 & 12,1 & 1,3 & 0,86 & 0,0919 & 0,0253 & 27,5 & 0,0666 & 0 & 0,0 & 0 & 0,0 & 1 & 99,6 \\
\hline 14,0 & 13,5 & 1,5 & 0,96 & 0,1026 & 0,0282 & 27,5 & 0,0744 & 0 & 0,0 & 0 & 0,0 & 1 & 91,4 \\
\hline 15,0 & 8,3 & 1,6 & 0,55 & 0,0728 & 0,0308 & 42,2 & 0,0420 & 0 & 0,0 & 0 & 0,0 & 1 & 85,9 \\
\hline 15,0 & 9,8 & 1,9 & 0,65 & 0,0860 & 0,0363 & 42,3 & 0,0497 & 0 & 0,0 & 0 & 0,0 & 1 & 75,7 \\
\hline 15,0 & 11,3 & 2,2 & 0,75 & 0,0992 & 0,0419 & 42,3 & 0,0573 & 0 & 0,0 & 1 & 94,0 & 1 & 67,9 \\
\hline 15,0 & 12,8 & 2,5 & 0,85 & 0,1124 & 0,0475 & 42,2 & 0,0649 & 0 & 0,0 & 1 & 85,6 & 1 & 61,6 \\
\hline 15,0 & 14,3 & 2,7 & 0,95 & 0,1256 & 0,0531 & 42,2 & 0,0725 & 0 & 0,0 & 1 & 78,8 & 2 & 95,9 \\
\hline 16,0 & 8,6 & 2,4 & 0,54 & 0,0870 & 0,0476 & 54,8 & 0,0394 & 0 & 0,0 & 1 & 88,0 & 1 & 63,8 \\
\hline 16,0 & 10,2 & 2,8 & 0,64 & 0,1031 & 0,0565 & 54,8 & 0,0467 & 1 & 99,7 & 1 & 77,5 & 2 & 94,3 \\
\hline 16,0 & 11,8 & 3,2 & 0,74 & 2 & 0,0 & 54,8 & 0,0539 & 1 & 89 & 1 & 69,6 & 2 & 84,6 \\
\hline 16,0 & 13,4 & 3,7 & 0,84 & 0,1353 & 0,0741 & 54,8 & 0,0612 & 1 & 81,4 & 1 & 63,3 & 2 & 77,0 \\
\hline 16,0 & 15,0 & 4,1 & 0,94 & 0,1514 & 0,0829 & 54,8 & 0,0685 & 1 & 74,8 & 2 & 97,7 & 3 & 95,9 \\
\hline 17,0 & 9,0 & 3,1 & 0,53 & 0,1026 & 0,0670 & 65,3 & 0,0356 & 1 & 91,8 & 1 & 71,4 & 2 & 86,9 \\
\hline 17,0 & 10,7 & 3,7 & 0,63 & 0,1220 & 0,0797 & 65,3 & 0,0423 & 1 & 80,7 & 1 & 62,7 & 2 & 76,3 \\
\hline 17,0 & 12,4 & 4,3 & 0,73 & 0,1414 & 0,0923 & 65,3 & 0,0490 & 1 & 72,2 & 2 & 94,3 & 3 & 92,6 \\
\hline 17,0 & 14,1 & 4,9 & 0,83 & 97 & 50 & 65,3 & 8 & 1 & 65,6 & 2 & 85,7 & 3 & 84,1 \\
\hline 17,0 & 15,8 & 5,5 & 0,93 & 0,1801 & 0,1176 & 65,3 & 0,0625 & 1 & 60,3 & 2 & 78,7 & 4 & 95,8 \\
\hline 18,0 & 9,3 & 3,9 & 0,52 & 0,1196 & 0,0883 & 73,8 & 0,0313 & 1 & 78,8 & 1 & 61,3 & 2 & 74,6 \\
\hline 18,0 & 11,1 & 4,6 & 0,62 & 0,1426 & 0,1053 & 73,8 & 0,0373 & 1 & 69,1 & 2 & 90,1 & 3 & 88,5 \\
\hline 18,0 & 12,9 & 5,4 & 0,72 & 0,1657 & 0,1223 & 73,8 & 0,0433 & 1 & 61,8 & 2 & 80,7 & 4 & 97,8 \\
\hline 18,0 & 14,7 & 6,1 & 0,82 & 0,1887 & 0,1393 & 73,8 & 0,0494 & 2 & 93,9 & 3 & 98,7 & 4 & 89,0 \\
\hline 18,0 & 16,5 & 6,9 & 0,92 & 0,2118 & 0,1564 & 73,8 & 0,0554 & 2 & 86,3 & 3 & 90,8 & 5 & 96,2 \\
\hline
\end{tabular}

Continua ... 
TABELA 4: Continuação ...

\begin{tabular}{|c|c|c|c|c|c|c|c|c|c|c|c|c|c|}
\hline \multirow{3}{*}{$\begin{array}{l}\text { DAP } \\
\mathrm{cm} \\
\end{array}$} & \multirow{3}{*}{$\begin{array}{l}\mathrm{h} \\
\mathrm{m}\end{array}$} & \multirow{3}{*}{$\begin{array}{c}\mathrm{h} 14 \\
\mathrm{~m} \\
\end{array}$} & \multirow[t]{3}{*}{$\mathrm{h} / \mathrm{d}$} & \multirow{3}{*}{$\begin{array}{c}\mathrm{v} 7 \\
\mathrm{~m}^{3} \mathrm{c} / \mathrm{c} \\
\end{array}$} & \multirow{3}{*}{$\begin{array}{c}\mathrm{v} 14 \\
\mathrm{~m}^{3} \mathrm{c} / \mathrm{c} \\
\end{array}$} & \multirow{3}{*}{$\begin{array}{l}\mathrm{v} 14 \\
\% \\
\end{array}$} & \multirow{3}{*}{$\begin{array}{l}\mathrm{v} 7-14 \\
\mathrm{~m}^{3} \mathrm{c} / \mathrm{c}\end{array}$} & \multicolumn{6}{|c|}{ Sortimento (v14) } \\
\hline & & & & & & & & \multicolumn{2}{|c|}{ S1 } & \multicolumn{2}{|c|}{ S2 } & \multicolumn{2}{|c|}{ S3 } \\
\hline & & & & & & & & $\mathrm{n}$ & $\%$ & $\mathrm{n}$ & $\%$ & $\mathrm{n}$ & $\%$ \\
\hline 19,0 & 9,7 & 4,6 & 0,51 & 0,1380 & 0,1109 & 80,4 & 0,0270 & 1 & 70,5 & 2 & 91,6 & 3 & 90,0 \\
\hline 19,0 & 11,6 & 5,5 & 0,61 & 0,1651 & 0,1328 & 80,4 & 0,0324 & 1 & 61,6 & 2 & 80,4 & 4 & 96,9 \\
\hline 19,0 & 13,5 & 6,4 & 0,71 & 0,1922 & 0,1546 & 80,4 & 0,0377 & 2 & 91,9 & 3 & 96,3 & 4 & 87,2 \\
\hline 19,0 & 15,4 & 7,3 & 0,81 & 0,2194 & 0,1764 & 80,4 & 0,0430 & 2 & 83,6 & 3 & 87,9 & 5 & 92,9 \\
\hline 19,0 & 17,3 & 8,2 & 0,91 & 0,2466 & 0,1982 & 80,4 & 0,0483 & 2 & 76,7 & 4 & 98,8 & 6 & 97,2 \\
\hline 20,0 & 9,9 & 5,2 & 0,50 & 0,1577 & 0,1345 & 85,3 & 0,0232 & 1 & 64,9 & 2 & 84,4 & 3 & 82,9 \\
\hline 20,0 & 11,9 & 6,2 & 0,60 & 0,1894 & 0,1615 & 85,3 & 0,0279 & 2 & 93,7 & 3 & 97,8 & 4 & 89,2 \\
\hline 20,0 & 13,9 & 7,3 & 0,70 & 0,2211 & 0,1886 & 85,3 & 0,0325 & 2 & 84,3 & 3 & 88,5 & 5 & 93,3 \\
\hline 20,0 & 15,9 & 8,3 & 0,80 & 0,2528 & 0,2156 & 85,3 & 0,0372 & 2 & 76,5 & 4 & 97,7 & 6 & 96,2 \\
\hline 20,0 & 17,9 & 9,4 & 0,90 & 0,2845 & 0,2427 & 85,3 & 0,0419 & 3 & 93,6 & 4 & 90,6 & 7 & 98,3 \\
\hline 21,0 & 10,2 & 5,8 & 0,49 & 0,1788 & 0,1588 & 88,8 & 0,0199 & 2 & 98,5 & 2 & 79,4 & 4 & 94,5 \\
\hline 21,0 & 12,3 & 7,0 & 0,59 & 0,2155 & 0,1915 & 88,8 & 0,0240 & 2 & 88,0 & 3 & 92,0 & 5 & 96,5 \\
\hline 21,0 & 14,4 & 8,1 & 0,69 & 0,2523 & 0,2241 & 88,8 & 0,0281 & 2 & 79,0 & 4 & 99,1 & 6 & 97,8 \\
\hline 21,0 & 16,5 & 9,3 & 0,79 & 0,2890 & 0,2568 & 88,8 & 0,0322 & 3 & 94,4 & 4 & 91,7 & 7 & 98,8 \\
\hline 21,0 & 18,6 & 10,5 & 0,89 & 0,3257 & 0,2894 & 88,9 & 0,0363 & 3 & 87,6 & 5 & 97,4 & 8 & 99,5 \\
\hline 22,0 & 10,5 & 6,3 & 0,48 & 0,2012 & 0,1839 & 91,4 & 0,0172 & 2 & 94,4 & 4 & 98,1 & 4 & 90,4 \\
\hline 22,0 & 12,7 & 7,6 & 0,58 & 0,2435 & 0,2226 & 91,4 & 0,0209 & 2 & 83,9 & 5 & 98,1 & 5 & 92,2 \\
\hline 22,0 & 14,9 & 8,9 & 0,68 & 0,2858 & 0,2613 & 91,4 & 0,0245 & 3 & 97,1 & 6 & 98,1 & 6 & 93,3 \\
\hline 22,0 & 17,1 & 10,2 & 0,78 & 0,3281 & 0,2999 & 91,4 & 0,0281 & 3 & 89,9 & 7 & 98,1 & 7 & 94,2 \\
\hline 22,0 & 19,3 & 11,6 & 0,88 & 0,3704 & 0,3386 & 91,4 & 0,0318 & 4 & 98,5 & 8 & 98,1 & 8 & 94,8 \\
\hline 23,0 & 10,7 & 6,7 & 0,46 & 0,2248 & 0,2098 & 93,3 & 0,0151 & 2 & 91,3 & 4 & 97,6 & 5 & 98,4 \\
\hline 23,0 & 13,0 & 8,2 & 0,56 & 0,2732 & 0,2549 & 93,3 & 0,0183 & 2 & 80,7 & 5 & 97,4 & 6 & 97,9 \\
\hline 23,0 & 15,3 & 9,7 & 0,66 & 0,3216 & 0,3000 & 93,3 & 0,0215 & 3 & 93,7 & 6 & 97,3 & 7 & 97,5 \\
\hline 23,0 & 17,6 & 11,1 & 0,76 & 0,3699 & 0,3451 & 93,3 & 0,0248 & 3 & 86,4 & 7 & 97,2 & 8 & 97,2 \\
\hline 23,0 & 19,9 & 12,6 & 0,86 & 0,4183 & 0,3903 & 93,3 & 0,0281 & 4 & 94,9 & 8 & 97,2 & 9 & 97,0 \\
\hline
\end{tabular}

Continua ...

Ciência Florestal, v. 9, n. 2, 1999 
TABELA 4: Continuação ...

\begin{tabular}{|c|c|c|c|c|c|c|c|c|c|c|c|c|c|}
\hline \multirow{3}{*}{$\begin{array}{l}\text { DAP } \\
\mathrm{cm}\end{array}$} & \multirow{3}{*}{$\begin{array}{l}\mathrm{h} \\
\mathrm{m}\end{array}$} & \multirow{3}{*}{$\begin{array}{c}\mathrm{h} 14 \\
\mathrm{~m} \\
\end{array}$} & \multirow[t]{3}{*}{$\mathrm{h} / \mathrm{d}$} & \multirow{3}{*}{$\begin{array}{c}\mathrm{v} 7 \\
\mathrm{~m}^{3} \mathrm{c} / \mathrm{c} \\
\end{array}$} & \multirow{3}{*}{$\begin{array}{c}\mathrm{v} 14 \\
\mathrm{~m}^{3} \mathrm{c} / \mathrm{c} \\
\end{array}$} & \multirow{3}{*}{$\begin{array}{l}\mathrm{v} 14 \\
\% \\
\end{array}$} & \multirow{3}{*}{$\begin{array}{l}\mathrm{v} 7-14 \\
\mathrm{~m}^{3} \mathrm{c} / \mathrm{c}\end{array}$} & \multicolumn{6}{|c|}{ Sortimento (v14) } \\
\hline & & & & & & & & \multicolumn{2}{|c|}{ S1 } & \multicolumn{2}{|c|}{ S2 } & \multicolumn{2}{|c|}{ S3 } \\
\hline & & & & & & & & $\mathrm{n}$ & $\%$ & $\mathrm{n}$ & $\%$ & $\mathrm{n}$ & $\%$ \\
\hline 24,0 & 10,9 & 7,2 & 0,45 & 0,2497 & 0,2364 & 94,7 & 0,0133 & 2 & 88,9 & 4 & 97,2 & 5 & 96,1 \\
\hline 24,0 & 13,3 & 8,7 & 0,55 & 0,3047 & 0,2884 & 94,7 & 0,0162 & 3 & 98,5 & 5 & 96,9 & 6 & 95,4 \\
\hline 24,0 & 15,7 & 10,3 & 0,65 & 0,3597 & 0,3405 & 94,7 & 0,0192 & 3 & 91,0 & 6 & 96,6 & 7 & 94,9 \\
\hline 24,0 & 18,1 & 11,9 & 0,75 & 0,4147 & 0,3925 & 94,7 & 0,0221 & 4 & 97,7 & 7 & 96,5 & 9 & 99,4 \\
\hline 24,0 & 20,5 & 13,5 & 0,85 & 0,4697 & 0,4446 & 94,7 & 0,0251 & 4 & 92,0 & 9 & 99,6 & 10 & 98,7 \\
\hline 25,0 & 11,1 & 7,5 & 0,44 & 0,2757 & 0,2638 & 95,7 & 0,0119 & 2 & 87,1 & 4 & 96,9 & 5 & 94,3 \\
\hline 25,0 & 13,6 & 9,2 & 0,54 & 0,3379 & 0,3233 & 95,7 & 0,0146 & 3 & 96,6 & 5 & 96,4 & 7 & 99,5 \\
\hline 25,0 & 16,1 & 10,9 & 0,64 & 0,4001 & 0,3828 & 95,7 & 0,0173 & 3 & 88,8 & 6 & 96,0 & 8 & 98,3 \\
\hline 25,0 & 18,6 & 12,6 & 0,74 & 0,4623 & 0,4423 & 95,7 & 0,0201 & 4 & 95,5 & 8 & 99,5 & 9 & 97,3 \\
\hline 25,0 & 21,1 & 14,3 & 0,84 & 0,5245 & 0,5018 & 95,7 & 0,0227 & 5 & 99,2 & 9 & 99,0 & 10 & 99,9 \\
\hline 26,0 & 11,2 & 7,9 & 0,43 & 0,3028 & 0,2919 & 96,4 & 0,0109 & 2 & 85,6 & 4 & 96,7 & 6 & 99,8 \\
\hline 26,0 & 13,8 & 9,7 & 0,53 & 0,3728 & 0,3594 & 96,4 & 0,0134 & 3 & 95,0 & 5 & 96,0 & 7 & 98,1 \\
\hline 26,0 & 16,4 & 11,5 & 0,63 & 0,4428 & 0,4269 & 96,4 & 0,0159 & 4 & 99,2 & 7 & 99,7 & 8 & 96,6 \\
\hline 26,0 & 19,0 & 13,3 & 0,73 & 0,5128 & 0,4944 & 96,4 & 0,0184 & 4 & 93,6 & 8 & 99,0 & 10 & 99,3 \\
\hline 26,0 & 21,6 & 15,1 & 0,83 & 0,5828 & 0,5619 & 96,4 & 0,0209 & 5 & 97,5 & 9 & 98,4 & 11 & 98,2 \\
\hline 27,0 & 11,4 & 8,1 & 0,42 & 0,3307 & 0,3208 & 97,0 & 0,0100 & 2 & 84,4 & 4 & 96,6 & 6 & 98,8 \\
\hline 27,0 & 14,1 & 10,1 & 0,52 & 0,4092 & 0,3968 & 97,0 & 0,0123 & 3 & 93,7 & 5 & 95,6 & 7 & 96,8 \\
\hline 27,0 & 16,8 & 12,0 & 0,62 & 0,4876 & 0,4729 & 97,0 & 0,0147 & 4 & 97,9 & 7 & 99,3 & 9 & 99,3 \\
\hline 27,0 & 19,5 & 13,9 & 0,72 & 0,5661 & 0,5490 & 97,0 & 0,0171 & 4 & 92,0 & 8 & 98,5 & 10 & 97,9 \\
\hline 27,0 & 22,2 & 15,9 & 0,82 & 0,6445 & 0,6251 & 97,0 & 0,0194 & 5 & 95,9 & 9 & 97,9 & 12 & 99,6 \\
\hline 28,0 & 11,5 & 8,4 & 0,41 & 0,3596 & 0,3503 & 97,4 & 0,0093 & 2 & 83,5 & 4 & 96,6 & 6 & 98,0 \\
\hline 28,0 & 14,3 & 10,4 & 0,51 & 0,4471 & 0,4356 & 97,4 & 0,0115 & 3 & 92,5 & 5 & 95,4 & 8 & 100 \\
\hline 28,0 & 17,1 & 12,5 & 0,61 & 0,5346 & 0,5208 & 97,4 & 0,0138 & 4 & 96,8 & 7 & 99,0 & 9 & 98,2 \\
\hline 28,0 & 19,9 & 14,5 & 0,71 & 0,6222 & 0,6061 & 97,4 & 0,0161 & 5 & 99,1 & 8 & 98,1 & 11 & 99,7 \\
\hline 28,0 & 22,7 & 16,5 & 0,81 & 0,7097 & 0,6914 & 97,4 & 0,0184 & 5 & 94,6 & 10 & 99,8 & 12 & 98,4 \\
\hline
\end{tabular}

Continua ... 
TABELA 4: Continuação ...

\begin{tabular}{|c|c|c|c|c|c|c|c|c|c|c|c|c|c|}
\hline \multirow{3}{*}{$\begin{array}{l}\text { DAP } \\
\mathrm{cm}\end{array}$} & \multirow{3}{*}{$\begin{array}{l}\mathrm{h} \\
\mathrm{m}\end{array}$} & \multirow{3}{*}{$\begin{array}{c}\mathrm{h} 14 \\
\mathrm{~m}\end{array}$} & \multirow[t]{3}{*}{$\mathrm{h} / \mathrm{d}$} & \multirow{3}{*}{$\begin{array}{c}\mathrm{v} 7 \\
\mathrm{~m}^{3} \mathrm{c} / \mathrm{c}\end{array}$} & \multirow{3}{*}{$\begin{array}{c}\mathrm{v} 14 \\
\mathrm{~m}^{3} \mathrm{c} / \mathrm{c}\end{array}$} & \multirow{3}{*}{$\begin{array}{l}\mathrm{v} 14 \\
\% \\
\end{array}$} & \multirow{3}{*}{$\begin{array}{l}\mathrm{v} 7-14 \\
\mathrm{~m}^{3} \mathrm{c} / \mathrm{c}\end{array}$} & \multicolumn{6}{|c|}{ Sortimento (v14) } \\
\hline & & & & & & & & \multicolumn{2}{|c|}{ S1 } & \multicolumn{2}{|c|}{ S2 } & \multicolumn{2}{|c|}{ S3 } \\
\hline & & & & & & & & $\mathrm{n}$ & $\%$ & $\mathrm{n}$ & $\%$ & $\mathrm{n}$ & $\%$ \\
\hline 29,0 & 11,6 & 8,6 & 0,40 & 0,3892 & 0,3804 & 97,7 & 0,0088 & 3 & 99,5 & 4 & 98,1 & 6 & 97,3 \\
\hline 29,0 & 14,5 & 10,7 & 0,50 & 0,4865 & 0,4755 & 97,7 & 0,0110 & 3 & 91,6 & 5 & 98,1 & 8 & 99,2 \\
\hline 29,0 & 17,4 & 12,9 & 0,60 & 0,5837 & 0,5706 & 97,8 & 0,0131 & 4 & 95,8 & 6 & 98,1 & 9 & 97,3 \\
\hline 29,0 & 20,3 & 15,0 & 0,70 & 0,6811 & 0,6657 & 97,7 & 0,0154 & 5 & 98,1 & 7 & 98,1 & 11 & 98,7 \\
\hline 29,0 & 23,2 & 17,2 & 0,80 & 0,7784 & 0,7608 & 97,7 & 0,0176 & 6 & 99,5 & 8 & 98,1 & 13 & 99,6 \\
\hline 30,0 & 11,7 & 8,8 & 0,39 & 0,4194 & 0,4110 & 98,0 & 0,0084 & 3 & 99,0 & 4 & 97,6 & 6 & 96,8 \\
\hline 30,0 & 14,7 & 11,0 & 0,49 & 0,5271 & 0,5166 & 98,0 & 0,0105 & 3 & 90,8 & 5 & 97,4 & 8 & 98,6 \\
\hline 30,0 & 17,7 & 13,3 & 0,59 & 0,6349 & 0,6223 & 98,0 & 0,0127 & 4 & 94,9 & 6 & 97,3 & 10 & 99,5 \\
\hline 30,0 & 20,7 & 15,5 & 0,69 & 0,7427 & 0,7279 & 98,0 & 0,0148 & 5 & 97,2 & 7 & 97,2 & 11 & 97,9 \\
\hline 30,0 & 23,7 & 17,8 & 0,79 & 0,8504 & 0,8335 & 98,0 & 0,0169 & 6 & 98,6 & 8 & 97,2 & 13 & 98,8 \\
\hline 31,0 & 11,7 & 8,9 & 0,38 & 0,4500 & 0,4420 & 98,2 & 0,0080 & 3 & 98,6 & 4 & 97,2 & 6 & 96,4 \\
\hline 31,0 & 14,8 & 11,3 & 0,48 & 0,5690 & 0,5588 & 98,2 & 0,0102 & 4 & 99,9 & 5 & 96,9 & 8 & 98,0 \\
\hline 31,0 & 17,9 & 13,6 & 0,58 & 0,6879 & 0,6757 & 98,2 & 0,0123 & 4 & 94,2 & 6 & 96,6 & 10 & 98,9 \\
\hline 31,0 & 21 , & 16,0 & 0,68 & 0,8 & 925 & 98,2 & 0,01 & 5 & 96,5 & 7 & 96,5 & 12 & 99,5 \\
\hline 31,0 & 24,1 & 18,3 & 0,78 & 0,9258 & 0,9093 & 98,2 & 0,0165 & 6 & 97,8 & 9 & 99,6 & 14 & 99,8 \\
\hline 32,0 & 11,8 & 9,0 & 0,37 & 0,4810 & 0,4732 & 98,4 & 0,0078 & 3 & 98,3 & 4 & 96,9 & 6 & 96,1 \\
\hline 32,0 & 15,0 & 11,5 & 0,47 & 0,6120 & 0,6020 & 98,4 & 0,0099 & 4 & 99,5 & 5 & 96,4 & 8 & 97,6 \\
\hline 32,0 & 18,2 & 13,9 & 0,57 & 0,7428 & 0,7308 & 98,4 & 0,0121 & 4 & 93,5 & 6 & 96,0 & 10 & 98,4 \\
\hline 32,0 & 21,4 & 16,4 & 0,67 & 0,8737 & 0,8596 & 98,4 & 0,0141 & 5 & 95,7 & 8 & 99,5 & 12 & 98,9 \\
\hline 32,0 & 24,6 & 18,9 & 0,77 & 1,0046 & 0,9883 & 98,4 & 0,0163 & 6 & 97,1 & 9 & 99,0 & 14 & 99,3 \\
\hline 33,0 & 11,8 & 9,1 & 0,36 & 0,5122 & 0,5046 & 98,5 & 0,0076 & 3 & 98,2 & 4 & 96,7 & 7 & 100 \\
\hline 33,0 & 15,1 & 11,7 & 0,46 & 0,6559 & 0,6460 & 98,5 & 0,0098 & 4 & 99,2 & 5 & 96,0 & 8 & 97,2 \\
\hline 33,0 & 18,4 & 14,2 & 0,56 & 0,7994 & 0,7875 & 98,5 & 0,0118 & 5 & 99,7 & 7 & 99,7 & 10 & 97,9 \\
\hline 33,0 & 21,7 & 16,8 & 0,66 & 0,9431 & 0,9290 & 98,5 & 0,0141 & 5 & 95,1 & 8 & 99,0 & 12 & 98,4 \\
\hline 33,0 & 25,0 & 19,4 & 0,76 & 1,0865 & 1,0705 & 98,5 & 0,0161 & 6 & 96,4 & 9 & 98,4 & 14 & 98,7 \\
\hline
\end{tabular}

Continua ...

Ciência Florestal, v. 9, n. 2, 1999 
TABELA 4: Continuação ...

\begin{tabular}{|c|c|c|c|c|c|c|c|c|c|c|c|c|c|}
\hline \multirow{3}{*}{$\begin{array}{l}\text { DAP } \\
\mathrm{cm}\end{array}$} & \multirow[b]{3}{*}{$\mathrm{m}$} & \multirow{3}{*}{$\begin{array}{c}\mathrm{h} 14 \\
\mathrm{~m}\end{array}$} & \multirow[t]{3}{*}{$\mathrm{h} / \mathrm{d}$} & \multirow{3}{*}{$\begin{array}{c}\mathrm{v} 7 \\
\mathrm{~m}^{3} \mathrm{c} / \mathrm{c} \\
\end{array}$} & \multirow{3}{*}{$\begin{array}{c}\mathrm{v} 14 \\
\mathrm{~m}^{3} \mathrm{c} / \mathrm{c} \\
\end{array}$} & \multirow{3}{*}{$\begin{array}{l}\mathrm{v} 14 \\
\%\end{array}$} & \multirow{3}{*}{$\begin{array}{l}\mathrm{v} 7-14 \\
\mathrm{~m}^{3} \mathrm{c} / \mathrm{c}\end{array}$} & \multicolumn{6}{|c|}{ Sortimento (v14) } \\
\hline & & & & & & & & \multicolumn{2}{|c|}{ S1 } & \multicolumn{2}{|c|}{ S2 } & \multicolumn{2}{|c|}{ S3 } \\
\hline & & & & & & & & $\mathrm{n}$ & $\%$ & $\mathrm{n}$ & $\%$ & $\mathrm{n}$ & $\%$ \\
\hline 34,0 & 11,8 & 9,2 & 0,35 & 0,5434 & 0,5359 & 98,6 & 0,0074 & 3 & 98,0 & 4 & 96,6 & 7 & 99,8 \\
\hline 34,0 & 15,2 & 11,8 & 0,45 & 0,7005 & 0,6908 & 98,6 & 0,0097 & 4 & 98,9 & 5 & 95,6 & 9 & 99,8 \\
\hline 34,0 & 18,6 & 14,5 & 0,55 & 0,8577 & 0,8458 & 98,6 & 0,0119 & 5 & 99,3 & 7 & 99,3 & 11 & 99,7 \\
\hline 34,0 & 22,0 & 17,2 & 0,65 & 1,0147 & 1,0007 & 98,6 & 0,0139 & 6 & 99,6 & 8 & 98,5 & 13 & 99,7 \\
\hline 34,0 & 25,4 & 19,8 & 0,75 & 1,1718 & 1,1557 & 98,6 & 0,0161 & 7 & 99,8 & 9 & 97,9 & 15 & 99,7 \\
\hline 35,0 & 11,7 & 9,2 & 0,34 & 0,5744 & 0,5670 & 98,7 & 0,0074 & 3 & 98,0 & 4 & 96,6 & 7 & 99,8 \\
\hline 35,0 & 15,2 & 12,0 & 0,44 & 0,7459 & 0,7363 & 98,7 & 0,0096 & 4 & 98,6 & 5 & 95,4 & 9 & 99,6 \\
\hline 35,0 & 18,7 & 14,7 & 0,54 & 0,9173 & 0,9055 & 98,7 & 0,0118 & 5 & 99,0 & 7 & 99,0 & 11 & 99,5 \\
\hline 35,0 & 22,2 & 17,5 & 0,64 & 1,0887 & 1,0747 & 98,7 & 0,0140 & 6 & 99,2 & 8 & 98,1 & 13 & 99,4 \\
\hline 35,0 & 25,7 & 20,2 & 0,74 & 1,2603 & 1,2439 & 98,7 & 0,0164 & 7 & 99,4 & 10 & 99,8 & 15 & 99,3 \\
\hline
\end{tabular}

Sendo: S1 = madeira para serraria com diâmetro na ponta fina superior a $14 \mathrm{~cm}$ com casca e comprimento de 2,8 metros; S2 = madeira para serraria com diâmetro na ponta fina superior a $14 \mathrm{~cm}$ com casca e comprimento de 2 metros; S3 = madeira para serraria com diâmetro na ponta fina superior a $14 \mathrm{~cm}$ com casca e comprimento de 1,30 metros. 